\title{
Shock propagation and diffraction through cavity
}

\author{
Arnab Chaudhuri \\ Department of Civil Engineering and Energy Technology \\ OsloMet - Oslo Metropolitan University, Oslo, Norway, arnab. chaudhuri@os lomet. no
}

\begin{abstract}
This work presents a numerical analysis of a planar moving shock wave with Mach number $M_{s}=1.3$, travelling through a square cavity geometry with rigid boundaries. A high-order artificial viscosity based Discontinuous Spectral Element Method (DSEM) is used for this purpose. The explicit numerical scheme utilizes entropy generation based transport coefficients to solve the conservative form of the viscous compressible fluid flow equations. Numerical prediction of the shock propagation and diffraction is found to be in excellent agreement with the experimental results of the literature. The stable numerical scheme resolves the detail of the complex flow dynamics for varying reference Reynolds number $\left(\mathrm{Re}_{\mathrm{f}}\right)$. The range of values of the artificial coefficients and the relative contribution of the components of the artificial energy dissipation rate are investigated and compared for different cases. Artificial energy dissipation is less for low $\mathrm{Re}_{\mathrm{f}}$. The dilatational dissipation dominates over other components till the incident shock wave resides in the flow domain.
\end{abstract}

Keywords: shock propagation, shock diffraction, shock reflection, DSEM, artificial viscosity

\section{Introduction}

Shock/Blast wave mitigation is an important research domain for disaster management. Shock wave propagation through solid structures are associated with complex shock-shock/shock-vortex/shock-boundary layer interactions. Understanding the fundamental features of shock wave propagation and diffraction is important for design of suitable mechanism for shock wave/ blast wave attenuation. Attenuation can be achieved by various means, e.g. utilising solid obstacles, foams, textiles, porous materials, granular filters, metallic grids, perforated plates, branched/bend duct, duct with rough walls, etc. Investigation related to shock/blast wave interaction with various configurations is consequently remaining as an active research field.

Apart from the experimental measurements, numerical approach can predict much more detailed flow evolution of such applications. Igra et al. summarizes both experimental and numerical works for many of these approaches (Igra et al., 2013). A detailed computational study of shock wave attenuation through solid obstacles are presented in (Chaudhuri et al., 2013). Application of abrupt changes in tunnel geometry is shown in (Igra et al., 1996,
1998, 2001). Most of the numerical simulations of shock diffraction problems of literature relied on inviscid simulations. However, viscous interactions become significant for shock-boundary layer/boundary layer-shear layer interactions and for long term shock-vortex dynamics. For example, the classical shock diffraction over $90^{\circ}$ sharp corner requires the consideration of no-slip walls in numerical approach to predict the secondary vortex and long-time evolution of flow dynamics (Sun and Takayama, 2003; Skews et al., 2012; Law et al., 2014; Chaudhuri and Jacobs, 2017).

It is desirable to use high-order numerical schemes together with stable shock capturing property to resolve the shocked flow dynamics. Numerical studies exploiting high-order schemes successfully reveal the general wave characteristics and complex flow dynamics of various phenomena associated with shock wave interaction with rigid bodies/boundaries like shock diffraction, reflection, focusing etc. (Chaudhuri et al., 2012; Glazer et al., 2011; Shadloo et al., 2014; Soni et al., 2017). The present work is aimed to perform viscous simulations and detailed analysis of shock propagation through the cavity like geometries/bend duct with cavities. A numerical analysis of a planar shock $\left(M_{s}=1.3\right)$ travelling through a two dimensional square cavity is carried out. A high-order artificial viscosity based DSEM is used for this purpose (Chaudhuri et al., 2017). A particular focus on the estimation of entropy generation based artificial viscosity coefficients and artificial viscous energy dissipation for various flow Reynolds number has been made.

The paper is organized as follows. A brief description of the governing equations is given in section 2 . The overall numerical approach is presented in section 3. In section 4, the problem setup for the present study is illustrated. Comparison of the findings with experimental results and flow analysis are discussed in section 5. Finally, conclusions are drawn in section 6.

\section{Governing equations}

Shock wave propagation through a gaseous medium is governed by the compressible Navier-Stokes (NS) system of equations. The non-dimensional form of the governing equations with artificial transfer coefficients is given by,

$$
\frac{\partial \mathbf{Q}}{\partial t}+\nabla \cdot \mathscr{F}^{\mathbf{a}}(\mathbf{Q})-\frac{1}{\operatorname{Re}_{\mathrm{f}}} \nabla \cdot \mathscr{F}^{\mathbf{v}}(\mathbf{Q})=0
$$


and

$$
\begin{array}{r}
\mathbf{Q}=\left(\begin{array}{c}
\rho \\
\rho \mathbf{v} \\
\rho E_{\mathrm{t}}
\end{array}\right), \mathscr{F} \mathbf{a}(\mathbf{Q})=\left(\begin{array}{c}
\rho \mathbf{v} \\
\rho \mathbf{v} \mathbf{v}+p \overline{\bar{\delta}} \\
\left(\rho E_{\mathrm{t}}+p \overline{\bar{\delta}}\right) \mathbf{v}
\end{array}\right), \\
\mathscr{F}^{\mathbf{v}}(\mathbf{Q})=\left(\begin{array}{c}
0 \\
\overline{\bar{\tau}} \\
\overline{\bar{\tau}} \mathbf{v}+\frac{\kappa_{\mathrm{eff}}}{(\gamma-1) M_{\mathrm{f}}^{2} \operatorname{Pr}_{\mathrm{f}}} \nabla T
\end{array}\right),
\end{array}
$$

where $\mathbf{Q}$ is the conservative solution vector, $\mathscr{F}^{\mathbf{a}}$ and $\mathscr{F} \mathbf{v}$ are the inviscid and viscous flux respectively. $\rho$ is the density, $\mathbf{v}$ is the velocity vector, and $E_{\mathrm{t}}$ is the total internal energy. $p$ is the static pressure and $T$ is the temperature. $\gamma$ is the ratio of specific heats. $\overline{\bar{\delta}}$ is the Kronecker delta tensor. Here, $M_{\mathrm{f}}, \operatorname{Re}_{\mathrm{f}}$ and $\mathrm{Pr}_{\mathrm{f}}$ are the reference Mach number, Reynolds number, and Prandtl number, respectively.

Assuming Stokes' hypothesis with zero bulk viscosity the viscous shear stress tensor can be written as, $\overline{\bar{\tau}}=2 \mu_{\text {eff }} \mathbf{S}-\frac{2}{3} \mu_{\text {eff }}(\nabla \cdot \mathbf{v}) \overline{\bar{\delta}}$, where $\mu_{\text {eff }}=\mu_{\mathrm{h}} \operatorname{Re}_{\mathrm{f}}+\mu$ is the effective dynamic viscosity and $\mathbf{S}=\frac{1}{2}\left[(\nabla \mathbf{v})^{\mathbf{T}}+\nabla \mathbf{v}\right]$ is the symmetric part of the velocity gradient tensor. The superscript " $T$ " designate a transpose. Similarly, $\kappa_{\text {eff }}=$ $\kappa_{\mathrm{h}} \mathrm{Re}_{\mathrm{f}}+\kappa$ is the effective thermal conductivity. Note that, $\mu_{\mathrm{h}}$ and $\kappa_{\mathrm{h}}$ are yet to be determined artificial transfer coefficients. For NS system of equations $\mu=\kappa=1$. In this study, $\gamma=1.4, M_{\mathrm{f}}=1$ and $\operatorname{Pr}_{\mathrm{f}}=0.72$ are prescribed. The ideal gas equation of state, $p=\rho T /\left(\gamma M_{\mathrm{f}}^{2}\right)$, closes Eqn. 1.

\section{Numerical approach}

The staggered Chebyshev collocation method is used to approximate the compressible NS system of equations with explicit time marching algorithms. In the nodal collocation formulation of the DSEM, the physical domain is subdivided with hexahedral physical elements. Each physical element is then mapped to a unit cubic computational element in the computational domain by an isoparametric transformation. The solution vector is collocated at the Chebyshev-Gauss quadrature points while the fluxes are collocated at the Chebyshev-Lobatto quadrature points. The detail of the discretization procedure can be found in (Kopriva, 1998; Jacobs et al., 2005).

The numerical scheme utilizes entropy generation based artificial transport coefficients to address Gibb's oscillation. Artificial viscosity coefficients are scaled with viscous and thermal entropy generation terms of the entropy transport equation. A shock sensor based switch is used to optimize the artificial coefficients. Also, the upper bound of artificial coefficients ensures that the inviscid time step remains smaller than the viscous time step. The basic idea of an artificial viscosity method is to explicitly add evenorder dissipation term to stabilize the scheme. However, artificial viscosity methods require arbitrary model constants as flow-dependent tuning parameters for the optimal solution. A brief description of the method of calculation of the artificial viscosity coefficients is presented here again for better clarity. The artificial momentum and thermal conductivity are linked with the viscous and conductive, entropy generating terms $\Phi$ and $\Gamma$ respectively. In non dimensional form this yields the following expression for the artificial viscosity coefficients: (Spurk and Aksel, 2008)

$$
\mu_{\mathrm{h}}=C_{\mu} \frac{\rho(\Delta h)^{2}}{\|\rho s-\overline{\rho s}\|_{\infty}}\left[\frac{\Phi}{T}\right]
$$

and

$$
\kappa_{\mathrm{h}}=C_{\kappa} \frac{\rho(\Delta h)^{2}}{\|\rho s-\overline{\rho s}\|_{\infty}}\left[\frac{1}{\operatorname{Pr}_{\mathrm{f}},(\gamma-1) M_{\mathrm{f}}^{2}} \frac{\Gamma}{T}\right],
$$

where $C_{\mu}$ and $C_{\kappa}$ are model parameters, $\Delta h$ is the mesh size, $\rho s=\frac{\rho}{\gamma(\gamma-1) M_{\mathrm{f}}^{2}} \ln \left(\frac{p}{\rho^{\gamma}}\right)$ and $\overline{\rho s}$ is the spatial average of $\rho s$. Here $\|\rho s-\overline{\rho s}\|_{\infty}$ is the globally computed supremum based on the global average entropy. The artificial viscosity coefficients defined above ensure the positivity (thus dissipative behavior) and $\mu_{\mathrm{h}}$ and $\kappa_{\mathrm{h}}$ scale with the grid spacing and vanish as $\Delta h \rightarrow 0$.

A shock sensor $\theta$ (Ducros et al., 1999) is used to control the artificial viscosity coefficients as $\mu_{\mathrm{h}} \theta \mathscr{H}(-\nabla \cdot \mathbf{v})$, and $\kappa_{\mathrm{h}} \theta$. This in turn reduces the dissipation in rotationdominated regions. The purpose of the Heaviside function $\mathscr{H}(-\nabla \cdot \mathbf{v})$ is to ensure that the dissipation is small in regions of isentropic expansion fans and contact discontinuities. The coefficients are controlled so that the inviscid time step $\Delta t_{\text {inv }}=\mathrm{CFL}_{\text {inv }} \Delta h /(|\mathbf{v}|+\sqrt{T})$, is smaller than the viscous time step. Here, $\mathrm{CFL}_{\text {inv }}$ is the corresponding CFL number for the inviscid time step. The upper limit of the artificial coefficient becomes $\mu_{\mathrm{h}, \mathrm{m}}=C_{\mathrm{m}} \rho \Delta h(|\mathbf{v}|+\sqrt{T})$, where $C_{\mathrm{m}} \propto \mathrm{CFL}_{\mathrm{vis}} / \mathrm{CFL}_{\text {inv }}$ represents another model parameter for the upper bound of $\mu_{\mathrm{h}}$. Note that, $\kappa_{\mathrm{h}}$ is also bounded by the $\mu_{\mathrm{h}, \mathrm{m}}$. The complete description of the scheme is reported in (Chaudhuri et al., 2017).

\section{Problem setup}

The geometry of the cavity flow configuration is taken similar to that presented in the experimental and the invis-

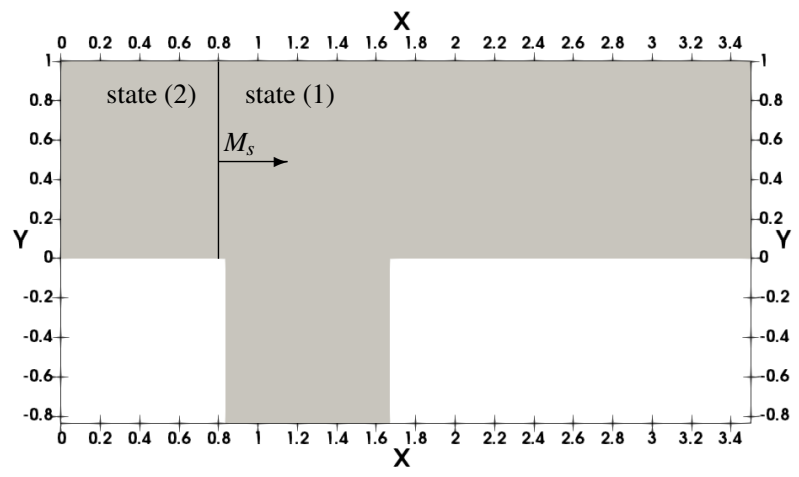

Figure 1. Schematic description of the cavity. 

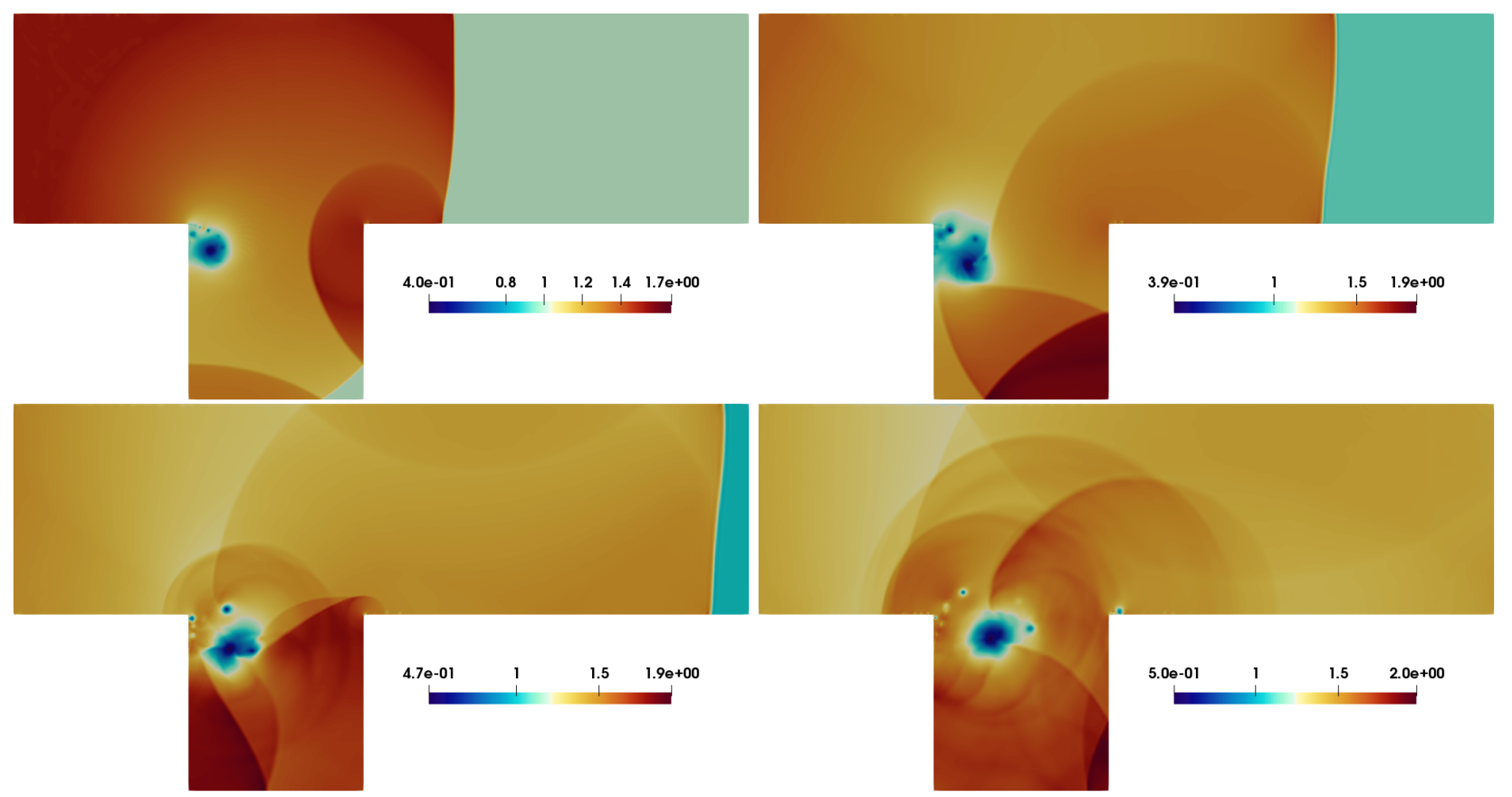

Figure 2. Density contours for $\mathrm{Re}_{\mathrm{f}}=10^{6}$, top left: $t=1.0$, top right: $t=1.5$, bottom left: $t=2.0$, and bottom right: $t=2.5$.

cid study of (Igra et al., 1996). Figure 1 shows the setup for the non-dimensional physical domain in the twodimensional (2D) $x-y$ plane. A shock wave with a Mach number $M_{\mathrm{S}}=1.3$ is allowed to pass through the cavity geometry. The initial shock location is at $x_{s}=0.8$ and the conditions are prescribed using the Rankine-Hugoniot relations for stagnant state (1) and shocked gas state (2). The left and right boundaries are set by the initial states. The other boundaries (top and bottom) are assigned with noslip wall conditions. The reference state $\phi_{\mathrm{f}}$, is taken as the stagnant state conditions $\phi_{1}$, to satisfy $M_{\mathrm{f}}=1$ and the reference length is taken as the height of the shock-tube of the experiment (Igra et al., 1996) $L_{\mathrm{f}}=60 \mathrm{~mm}$. 50,000 P3 (fourth order) elements with a total of $0.8 \times 10^{6}$ degrees of freedom are considered in the domain. In the present study, the value of the CFL number is taken as 0.9 and the model constants for artificial viscosity are set as: $\left(C_{\mu}, C_{\kappa}, C_{\mathrm{m}}\right)=(0.5,0.25,0.15)$ like our previous studies. A very weak filter is used, filter order of 64 (Chaudhuri et al., 2017) to enhance the stability of the method. The simulations are performed on Supermicro X9DRT compute nodes (dual Intel E5-2670). A typical test case utilizes 12 nodes consuming about $860 \mathrm{CPU}$ hours.

\section{Results and Discussion}

In subsection 5.1, first a discussion the flow evolution associated with the shock propagation and diffraction through the cavity geometry will be given. The analysis of artificial viscosity based DSEM methodology in light of the artificial dissipation will be given in the following subsection 5.2. Simulations are carried out with varying $\mathrm{Re}_{\mathrm{f}}$, namely $10^{6}, 10^{5}$, and $10^{4}$. The same mesh in all cases has been used.

\subsection{General flow evolution}

Figure 2 shows the density contours for $\mathrm{Re}_{\mathrm{f}}=10^{6}$ at different time instants. The incident shock (IS) is first gets diffracted in the left corner of the cavity. Subsequently, the corner primary vortex and shear layer instability evolves while incident shock propagates further downstream. The primary vortex grows and moves away from the corner of the cavity. The secondary vortices arising due to viscous effects on the no-slip wall, interact with shear layer and influence in the evolution of the unstable shear layer. This secondary vortex interaction is similar in nature to those reported for classical $M_{s}=1.5$ flow over $90^{\circ}$ diffraction corner, see e.g. (Sun and Takayama, 2003; Skews et al., 2012; Law et al., 2014; Chaudhuri and Jacobs, 2017). The incident shock further gets diffracted in the right corner of the cavity and produces the reflected wave which rebounds back and forth in the cavity section and intensely perturbs the growing primary vortex and the shear layer. Several transverse waves arise from the multiple reflections/diffractions within the cavity and the top wall of the flow configuration. The incident shock leaves the domain at $t \approx 2.1$. Note that, the present viscous simulations capture the viscous effects of no-slip boundary interaction, together with the complex shock dynamics. To resolve these, refined elements are generated using standard stretching functions (Chung, 2010) near the wall regions. Artificial viscosity based DSEM scheme is capable of resolving all the features of the complex flow dynamics.

Figure 3a shows the evolution of the pressure profile along the flow direction at $y=0.5$. The initial pressure jump associated with $M_{s}=1.3$ is $P_{2} / P_{1}=1.805$. Incident shock propagates with inviscid speed while leaving the domain boundary with a little lower intensity of $P / P_{1} \approx 1.68$. The 

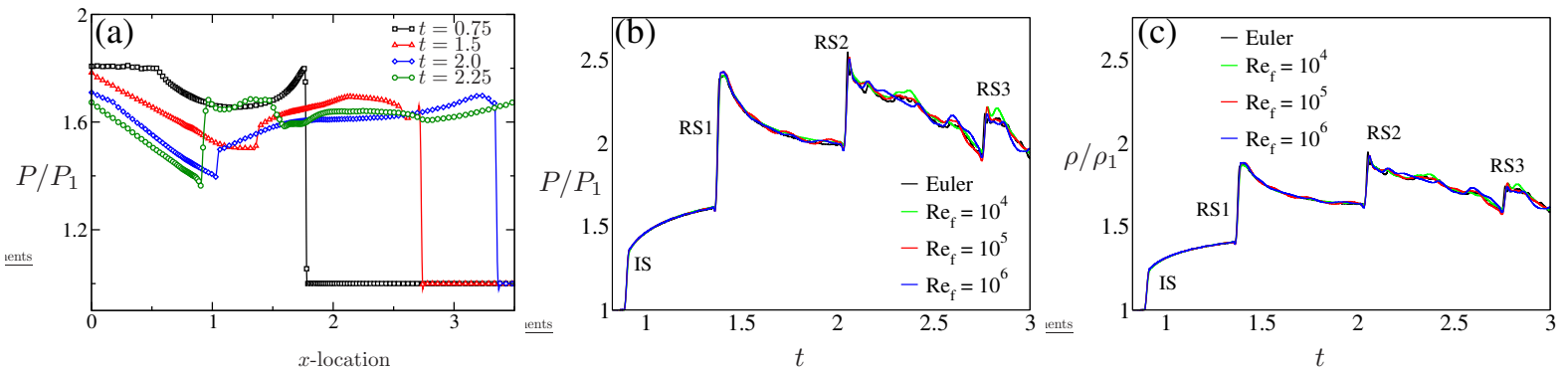

Figure 3. (a): Pressure evolution for $\operatorname{Re}_{\mathrm{f}}=10^{6}$ at $y=0.5$, (b) and (c): probe signals for different cases.
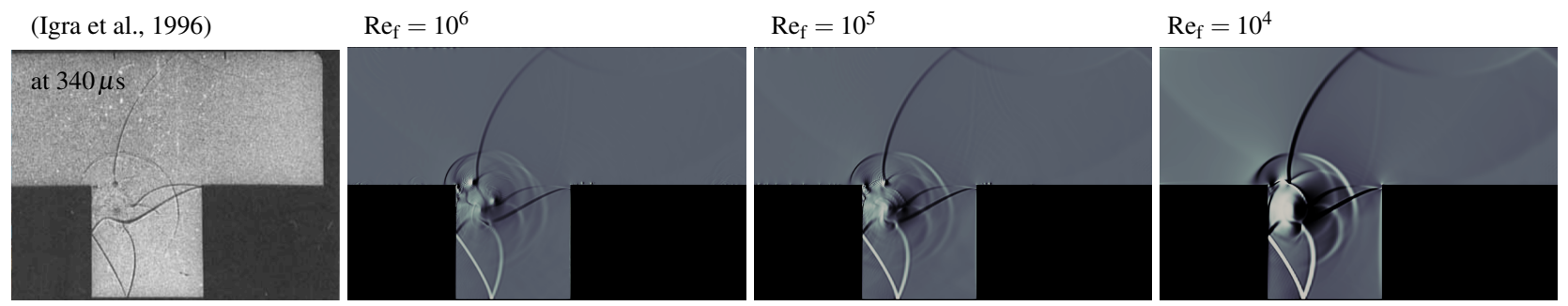

Figure 4. Comparison of experimental Shadowgraph (Igra et al., 1996) with numerical Schlieren at $t=1.9$.

propagation and diffraction through the cavity geometry exhibit an attenuation effect. Flow quantities are recorded at a probe location situated at the middle of the bottom wall of the cavity. Figures $3 b$ and $3 c$ show the pressure and density evolution at this probe location. The signature of the reflected waves passing through this probe is clearly seen in this figure. The signals show the incident shock and several reflected shocks (RS's).The RS1 from the right wall of the cavity bounces between the cavity walls and produces the subsequent RS's. The pressure peaks agree very well with the experimental and the inviscid numerical work of (Igra et al., 1996; Igra and Igra, 2016). Note that, this shock dynamics remains essentially similar irrespective of the $\mathrm{Re}_{\mathrm{f}}$. This is expected, as the shock dynamics is very fast compare to the slow viscous effects. Only little variations are observed once the incident shock is out of the domain. The overall shock dynamics are found to be in excellent agreement with the experimental (Igra et al., 1996) results (see Figure 4). Based on reference length $L_{f}=60 \mathrm{~mm}$, the $R_{\mathrm{f}}$ for the experimental setup of (Igra et al., 1996) lies in the range of $10^{6}$. Figure 4 clearly depicts that the experimental shadowgraph is essentially similar to the numerical Schlieren pictures. The resemblance is most for $\mathrm{Re}_{\mathrm{f}}=10^{6}$. For lower $\mathrm{Re}_{\mathrm{f}}$ viscous effects stabilize the shear layer and the secondary vortex interaction is less dramatic compared to the cases with higher $\mathrm{Re}_{\mathrm{f}}$. Evidently, the shock structures are much thicker for $\operatorname{Re}_{\mathrm{f}}=10^{4}$.

\subsection{Artificial dissipation of DSEM}

In the artificial viscosity based DSEM formulation $\mu_{\mathrm{h}}$ and $\kappa_{\mathrm{h}}$ are calculated explicitly (see section 3 ). These are designed to achieve optimal dissipation in shock dominated regions of the flow-field by the use of a shock sensor and the Heaviside function (see section 3). Figure 5 shows that the range of values of $\mu_{\mathrm{h}}$ (see left column) remains similar in all cases. The contribution of $\mu_{\mathrm{h}}$ and $\kappa_{\mathrm{h}}$ predominantly lie in the regions with shocks/shocklets. The contours are in accordance with the shock structure of the flow-field. For higher $\mathrm{Re}_{\mathrm{f}}$, one can notice shock thicknesses are much less (in accordance with Figure 4). The contours of the local mesh Reynolds number, $\mathrm{Re}_{\mathrm{h}}$ (defined as $\operatorname{Re}_{\mathrm{h}}=\Delta h|\mathbf{v}| \rho \operatorname{Re}_{\mathrm{f}}$ ) are also shown in Figure 5. Note that, here the mesh remains the same for three cases. These contours look very similar in nature with expected difference in order of magnitude. For $\operatorname{Re}_{\mathrm{f}}=10^{6}$, the $\mathrm{Re}_{\mathrm{h}} \lesssim 2800$, for $\mathrm{Re}_{\mathrm{f}}=10^{5}$, the $\mathrm{Re}_{\mathrm{h}} \lesssim 280$ and for $\mathrm{Re}_{\mathrm{f}}=10^{4}$, the $\mathrm{Re}_{\mathrm{h}} \lesssim 26$. As mentioned in (Chaudhuri et al., 2017; Chaudhuri and Jacobs, 2017), the inverse of the $\mu_{\mathrm{h}}$ is a measure of the local effective Reynolds number. In previous studies, it is observed that, for high $\mathrm{Re}_{\mathrm{f}}$ the maximum of the added artificial viscosity lies in the order of the inverse of the local $\mathrm{Re}_{\mathrm{h}}$. This is being illustrated in the contours of $\mu_{\mathrm{h}} \mathrm{Re}_{\mathrm{h}}$ in Figure 5. For cases with lower $\operatorname{Re}_{\mathrm{f}}$ the maximum of the $\mu_{\mathrm{h}} \mathrm{Re}_{\mathrm{h}}$ gets reduced. This clearly depicts the fact that the added dissipation is optimal in all cases. The relative contribution of artificial dissipation is less for low $R_{f}$. Note that the range of values of $\kappa_{\mathrm{h}}$ always remains smaller than $\mu_{\mathrm{h}}$ (not shown). The artificial viscosity based DSEM is thus capable of adding a controllable amount of dissipation for capturing shocks and yields stable solutions.

A further investigatation of the components of the artificial energy dissipation rate associated with the transport equation of the instantaneous kinetic energy. The energy dissipation rate $\mathscr{E}$ associated with $\mu_{\mathrm{h}}$ consists of rotational $\mathscr{E}_{\mathrm{r}}$, non-homogeneous $\mathscr{E}_{\mathrm{nh}}$ and dilatation dissipations $\mathscr{E}_{\mathrm{d}}$ as:

$$
\mathscr{E}=\mathscr{E}_{\mathrm{r}}+\mathscr{E}_{\mathrm{nh}}+\mathscr{E}_{\mathrm{d}}
$$



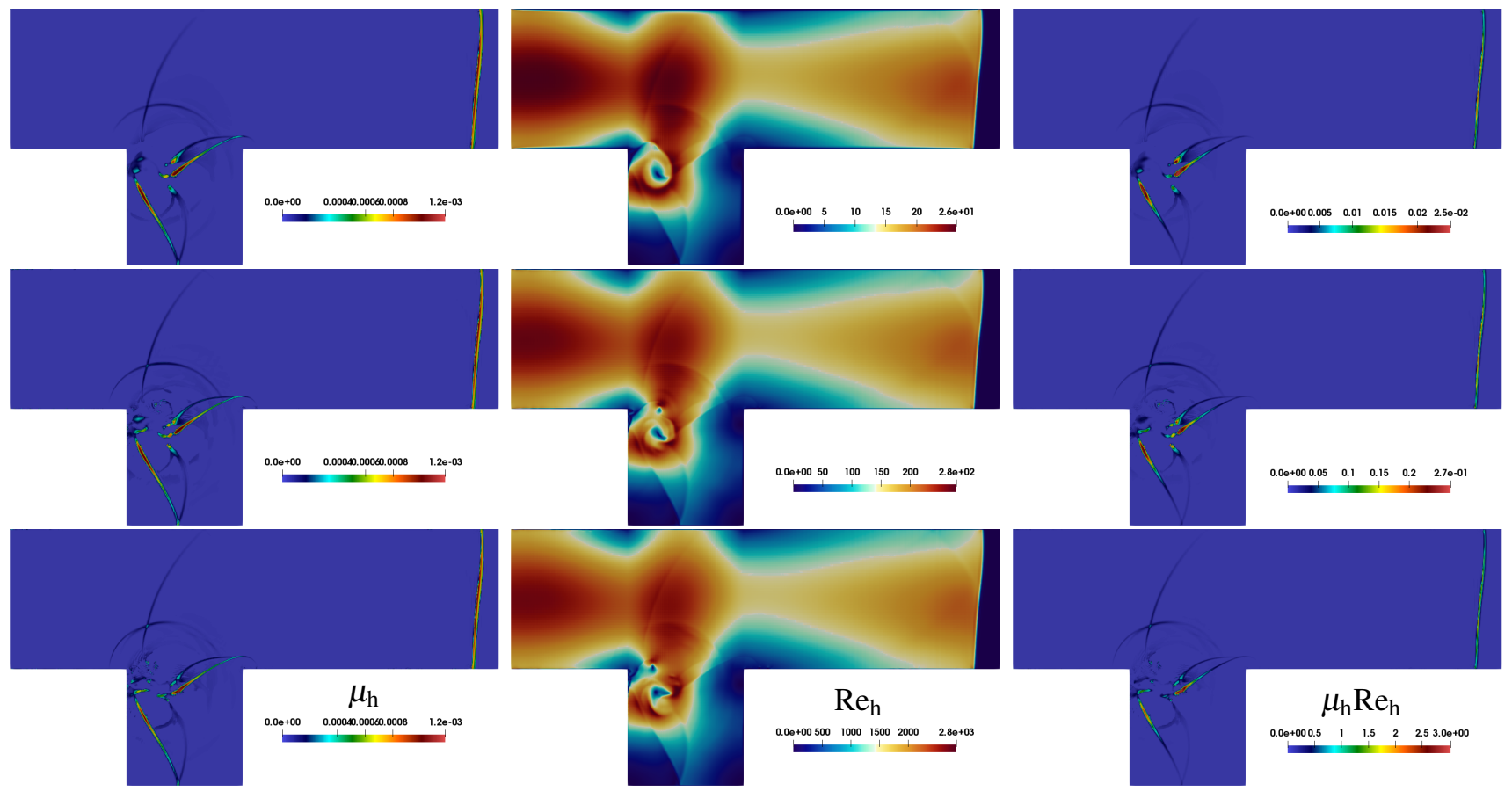

Figure 5. Contours of $\mu_{\mathrm{h}}, \operatorname{Re}_{\mathrm{h}}$ and $\mu_{\mathrm{h}} \operatorname{Re}_{\mathrm{h}}$ at $t=2$, top row: $\operatorname{Re}_{\mathrm{f}}=10^{4}$, middle row: $\operatorname{Re}_{\mathrm{f}}=10^{5}$ and bottom row: $\operatorname{Re}_{\mathrm{f}}=10^{6}$.
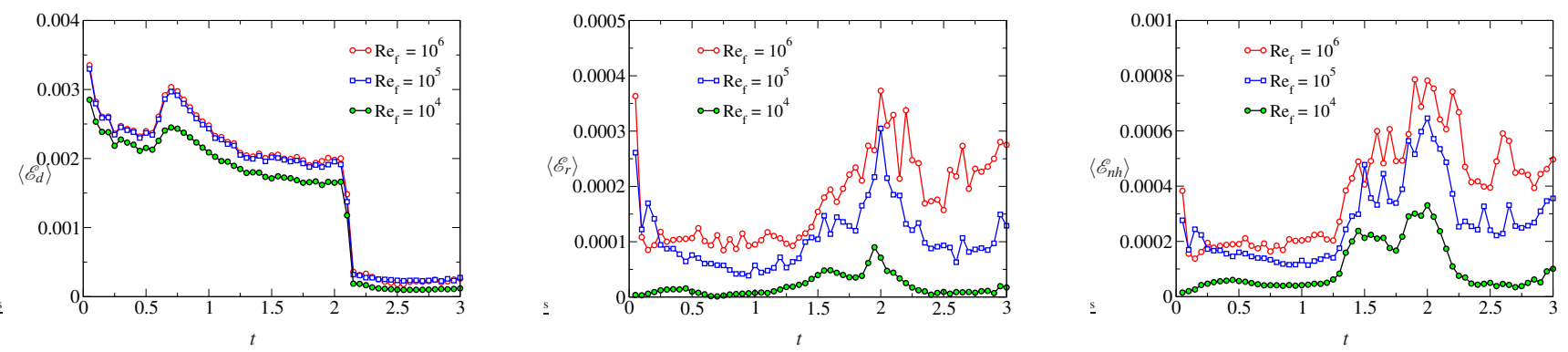

Figure 6. Components of the artificial energy dissipation rate.

where, $\mathscr{E}_{\mathrm{r}}=\mu_{\mathrm{h}} \Omega, \mathscr{E}_{\mathrm{nh}}=2 \mu_{\mathrm{h}}\left[(\nabla \mathbf{v})^{\mathbf{T}} \nabla \mathbf{v}-(\nabla \cdot \mathbf{v})^{\mathbf{2}}\right]$, and $\mathscr{E}_{\mathrm{d}}=\frac{4}{3} \mu_{\mathrm{h}}(\nabla \cdot \mathbf{v})^{2}$. Here, $\Omega$ is the enstrophy. Figure 6 shows the area-weighted (entire domain) dissipation rates as a function of time. For any property $\phi$, the area weighting is defined as $\langle\phi\rangle=\frac{\int \phi d A}{\int d A}$. It can be seen that $\langle\mathscr{E}\rangle$ is lower for lower $\mathrm{Re}_{\mathrm{f}}$. Each contributing term follows the same behavior. Clearly, the dominant contributing term for total kinetic energy dissipation rate is $\mathscr{E}_{\mathrm{d}}$ till the incident shock remains in the flow domain i.e., $t \lesssim 2.1$. Evidently, the component terms become comparable for $t>2.1$ (see Figure 7 for different $\left.\operatorname{Re}_{\mathrm{f}}\right)$. One can notice that the, $\left\langle\mathscr{E}_{n h}\right\rangle$ becomes significant in all cases $\approx 30 \%-50 \%$. On the other hand, $\mathscr{E}_{\mathrm{r}}$ remains $\lesssim 20 \%$ in all cases. Note that, the values of $\langle\mathscr{E}\rangle$ remain typically two-order of magnitude lower than the pressure dilatational term as found in (Chaudhuri and Jacobs, 2017).

\section{Conclusions}

In this work a study of shock propagation and diffraction of a planar shock $\left(M_{s}=1.3\right)$ travelling through a square cavity is performed. The geometry is taken similar to a previous literature. An artificial viscosity based DSEM is used for this purpose. The findings can be summarized as:

- The study with 50,000 P3 (fourth order) elements resolved all flow features well. The results are in excellent agreement with experimental results of literature. Accounting the viscous effects, the boundary and shear layer interaction with shock wave is well predicted by the present simulations. The pressure signal at the mid location of the bottom wall of the cavity shows multiple shock reflections on the cavity walls. The signals are similar irrespective of $\operatorname{Re}_{\mathrm{f}}$. The pressure peaks agree very well with (Igra et al., 1996) and the recent work (Igra and Igra, 2016).

- Estimation of artificial viscosity coefficients for various $\mathrm{Re}_{\mathrm{f}}$ reveals the consistency of the entropy generation based formulation. The time evolutions of area weighted average quantities of the components of the artificial energy dissipation rate are in accordance of the shocked flow physics.

Dissipations dictated by entropy generation in the pre- 

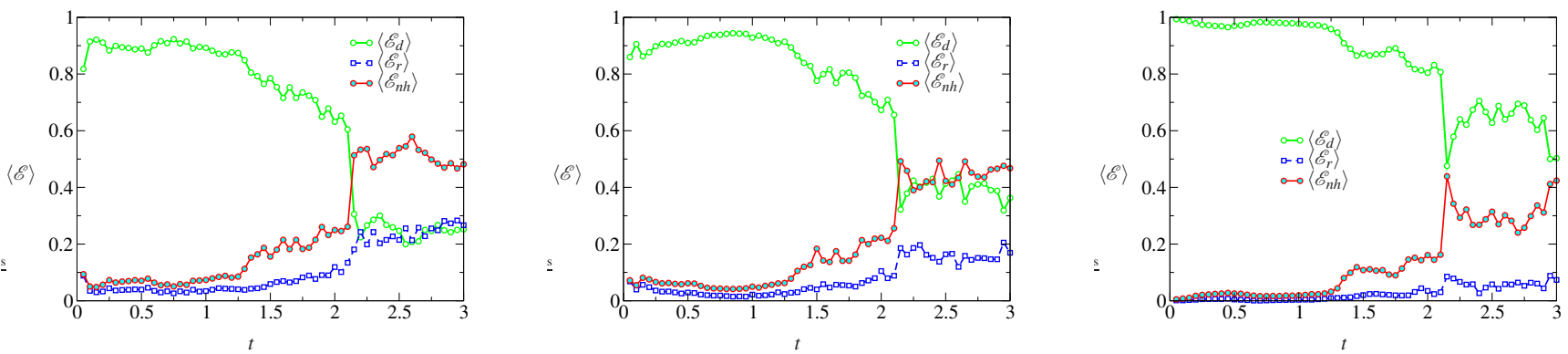

Figure 7. Normalized components of the artificial energy dissipation rate, left: $\operatorname{Re}_{\mathrm{f}}=10^{6}$, middle: $\operatorname{Re}_{\mathrm{f}}=10^{5}, \mathrm{right}_{\mathrm{R}} \mathrm{Re}_{\mathrm{f}}=10^{4}$.

sent artificial viscosity based methodology in a way account the effects of turbulence models. In the future, studies with 3D Large eddy simulations will be performed for further, deeper analysis related to the role of the artificial viscosity coefficients.

\section{Acknowledgement}

This work utilized the supercomputing facility of Abel computer cluster (University of Oslo) under the project NN9542K at Notur, UNINETT Sigma2 AS (National infrastructure for scientific computational science in Norway). The author greatly acknowledges the collaboration with G.B. Jacobs of San Diego State University, W. S. Don of Ocean University of China and F. Mashayek of University of Illinois at Chicago.

\section{References}

A Chaudhuri and GB Jacobs. Dynamics of shock wave diffraction over sharp splitter geometry using entropy-based artificial viscosity method. Shock Waves, pages 1-15, 2017.

A Chaudhuri, A Hadjadj, O Sadot, and E Glazer. Computational study of shock-wave interaction with solid obstacles using immersed boundary methods. International Journal for Numerical Methods in Engineering, 89(8):975-990, 2012. doi:https://doi.org/10.1002/nme.3271.

A Chaudhuri, A Hadjadj, O Sadot, and G Ben-Dor. Numerical study of shock-wave mitigation through matrices of solid obstacles. Shock Waves, 23(1):91-101, 2013. doi:https://doi.org/10.1007/s00193-012-0362-2.

A. Chaudhuri, G.B. Jacobs, W.S. Don, H. Abbassi, and F. Mashayek. Explicit discontinuous spectral element method with entropy generation based artificial viscosity for shocked viscous flows. Journal of Computational Physics, 332:99 - 117, 2017. ISSN 0021-9991. doi:http://dx.doi.org/10.1016/j.jcp.2016.11.042. URL http://www.sciencedirect.com/science/ article/pii/s0021999116306349.

TJ Chung. Computational fluid dynamics. Cambridge university press, 2010.

F Ducros, V Ferrand, Franck Nicoud, C Weber, D Darracq, C Gacherieu, and Thierry Poinsot. Large-eddy simulation of the shock/turbulence interaction. Journal of Computational Physics, 152(2):517-549, 1999. doi:https://doi.org/10.1006/jcph.1999.6238.
E Glazer, O Sadot, A Hadjadj, and A Chaudhuri. Velocity scaling of a shock wave reflected off a circular cylinder. Physical Review E, 83(6):066317, 2011.

Dan Igra and Ozer Igra. Planar shock-wave diffraction into a square cavity filled with different gases. AIAA Journal, pages 1798-1802, 2016.

O Igra, J Falcovitz, H Reichenbach, and W Heilig. Experimental and numerical study of the interaction between a planar shock wave and a square cavity. Journal of Fluid Mechanics, 313: 105-130, 1996.

O Igra, L Wang, J Falcovitz, and W Heilig. Shock wave propagation in a branched duct. Shock Waves, 8(6):375-381, 1998.

O Igra, X Wu, J Falcovitz, T Meguro, K Takayama, and W Heilig. Experimental and theoretical study of shock wave propagation through double-bend ducts. Journal of Fluid Mechanics, 437:255-282, 2001.

O Igra, J Falcovitz, L Houas, and G Jourdan. Review of methods to attenuate shock/blast waves. Progress in Aerospace Sciences, 58:1-35, 2013.

Gustaaf B Jacobs, David A Kopriva, and Farzad Mashayek. Validation study of a multidomain spectral code for simulation of turbulent flows. AIAA Journal, 43(6):1256-1264, 2005.

David A Kopriva. A staggered-grid multidomain spectral method for the compressible Navier-Stokes equations. Journal of Computational Physics, 143(1):125-158, 1998.

C Law, AO Muritala, and BW Skews. Unsteady flow with separation behind a shock wave diffracting over curved walls. Shock Waves, 24(3):283-294, 2014. doi:https://doi.org/10.1007/s00193-013-0486-z.

MS Shadloo, A Hadjadj, and A Chaudhuri. On the onset of postshock flow instabilities over concave surfaces. Physics of Fluids (1994-present), 26(7):076101, 2014. doi:http://dx.doi.org/10.1063/1.4890482.

Beric Skews, Craig Law, Adam Muritala, and Sebastian Bode. Shear layer behavior resulting from shock wave diffraction. Experiments in fluids, 52(2):417-424, 2012. doi:https://doi.org/10.1007/s00348-011-1233-9.

V Soni, A Hadjadj, A Chaudhuri, and G Ben-Dor. Shock-wave reflections over double-concave cylindrical reflectors. Journal of Fluid Mechanics, 813:70-84, 2017. 
Joseph H. Spurk and Nuri Aksel. Fluid Mechanics. SpringerVerlag Berlin Heidelberg, 2008. ISBN 978-3-540-73537-3. doi:https://doi.org/10.1007/978-3-540-73537-3.

M Sun and K Takayama. A note on numerical simulation of vortical structures in shock diffraction. Shock Waves, 13(1): 25-32, 2003. doi:https://doi.org/10.1007/s00193-003-01950. 